\title{
POBREZA E INEQUIDAD: LA HISTORIA OFICIAL
}

\section{Henry Veltmeyer* \\ (Traducción de Luis Rodolfo Morán Quiroz)}

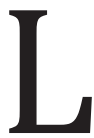
a Declaración del Milenio de la Organización de Naciones Unidas (ONU), adoptada por la Asamblea General en su Resolución 55/2 del 8 de septiembre de 2000, significó un hito para la corriente dominante de los estudios del desarrollo. La pobreza había sido vista durante mucho tiempo como la pieza clave de la estrategia del desarrollo, pero no fue sino hasta finales de la última década del siglo XX, después de una década y media de políticas neoliberales bajo el llamado Consenso de Washington, que la comunidad internacional del desarrollo logró al fin comprender o aceptar, al menos en parte, la idea promulgada por académicos de que la pobreza era sistémica, resultado de los modos de operar del sistema y del lugar que los pobres ocupen en éste, y no un fracaso de los mismos pobres; y que por tanto requería una perspectiva más sistémica sobre el desarrollo.

El discurso dominante no propone un cambio en el sistema subyacente (capitalismo) como tal, sino un marco institucional y de políticas completamente nuevo, un nuevo paradigma de desarrollo: un desarrollo socialmente incluyente (en la formulación de los teóricos de la Comisión Económica para América Latina y el Caribe [Cepal], Osvaldo Sunkel y Roberto Infante).

Como lo describieron los economistas del Departamento de Asuntos Económicos y Sociales (DAES, 2010) de la ONU en un estudio más reciente, los Objetivos de Desarrollo del Milenio (ODM), acordados por la comunidad internacional en 2000, "encarnaban un redescubrimiento de la reflexión de que las estrategias de crecimiento basadas en el mercado eran insuficientes por sí mismas para resolver el problema de la pobreza generalizada y que se

* Profesor-investigador del Doctorado en Estudios del Desarrollo de la Universidad Autónoma de Zacatecas y Director de la Red de Estudios Críticos del Desarrollo. 
requerían instituciones con un funcionamiento adecuado y políticas sociales efectivas para asegurar una adecuada provisión de servicios de salud y educación, y para evitar la exclusión social de muchas personas".

Detrás de esta "reflexión" subyace el Posconsenso de Washington en el sentido de que la pobreza no sólo estaba arraigada en las estructuras sociales e institucionales del sistema - la "estructura de inequidad social", según el medular informe de CEPAL de 2010 intitulado La hora de la igualdad. Brechas por cerrar, caminos por abrir - sino que las políticas neoliberales de "libre mercado", exigidas y puestas en práctica bajo el Consenso de Washington, eran responsables de generar nuevas formas de inequidad social y de pobreza. El Programa de las Naciones Unidas para el Desarrollo (PNUD), en un informe paralelo, llegó precisamente a la misma conclusión. En palabras de este informe, existe una "correspondencia directa entre el avance de la globalización, el neoliberalismo y el avance de la inequidad social, la pobreza y la desigualdad social. Las contradicciones más explosivas", añade el informe, "se dan porque el avance de la globalización [neoliberal] marcha de la mano del avance de la pobreza y la polarización social" (PNUD, 2010: XV). Y, continúa el informe, "es innegable que los años ochenta y noventa [constituyeron] la creación de una diferencia abismal entre la riqueza y la pobreza", y que esta diferencia constituye el obstáculo más formidable para el logro del desarrollo humano (PNUD, 2010: XV).

Un cambio importante respecto a la manera en que se concebía y abordaba la pobreza bajo la égida del Consenso de Washington se refleja en el cambio de un debate en torno a la injusticia (unfairness) en la distribución de la riqueza y el ingreso hacia una preocupación por un desarrollo socialmente incluyente, para asegurar la participación activa de los pobres y de los "excluidos hasta el momento" (unos cuatro mil millones, según la International Finance Corporación del Banco Mundial) en el proceso de producción, los beneficios de la globalización y los servicios gubernamentales vitales (Sunkel e Infante, 2009; ONU, 2005; UNDESA, 2010). Esta 
perspectiva - que vincula la pobreza con la exclusión, y que ve la pobreza como la subutilización de las oportunidades de empredurismo, en vez de verla cómo una tragedia, y la inequidad como fuente de malestar social y potencial desestabilización - ya había despertado el entusiasta apoyo de parte de la comunidad internacional del desarrollo para los estudios de Hernando de Soto sobre la capitalización de los pobres mediante el otorgamiento de títulos legales de su tierra y la esperanza de transformar a los pobres, si no en empresarios, al menos en consumidores (ONU, 2005: 50). Dentro de las agencias operativas del sistema de la ONU, empero, el nuevo consenso adoptó la forma de un papel más intervencionista para los gobiernos, asignándoles la responsabilidad de asumir acciones - con la cooperación internacional y la participación social- para asegurar con ello la realización de los ODM (reducción de la pobreza, etcétera) dentro del marco institucional y de políticas del "nuevo desarrollismo" (desarrollo socialmente incluyente).

Esta nueva perspectiva incluía, y se basaba en, un nuevo "marco amplio de desarrollo" (diseñado en el Banco Mundial bajo la presidencia de Wolfensohn) y un nuevo instrumento de políticas de desarrollo, el Documento de estrategia de reducción de la pobreza (DERP, o PRSP, por sus siglas en inglés). Dentro de este marco, los esfuerzos de desarrollo de los gobiernos y las organizaciones internacionales se han enfocado en la reducción de la pobreza específicamente, el objetivo número 1: reducir a la mitad la tasa de pobreza extrema para 2015- por medio de un enfoque del desarrollo más incluyente de parte de un Estado posneoliberal más activo y por medio de transferencias directas de dinero del gobierno hacia los pobres - una política iniciada en Honduras y Brasil, pero que en la actualidad ha sido adoptada por unos 19 países en América latina.

Algunos de los rasgos interesantes y sobresalientes de este cambio en las políticas públicas y en la nueva perspectiva estratégica y política para la reducción de la pobreza en el nuevo milenio incluyen los vínculos entre este enfoque y los patrones de cambio observados (y hasta cierto punto estudiados) sobre la 
"problemática de la pobreza" y lo que la ONU define como el "predicamento de la inequidad" (su potencial para generar malestar social e inestabilidad política). ${ }^{1}$ Una serie de informes oficiales han documentado un patrón de reducción significativa de la pobreza y una convergencia global respecto a la distribución de la riqueza y los ingresos. Sin embargo, si estas mejoras reflejan cambios en la economía global, un nuevo desarrollismo u otros factores, es poco claro. La ONU, en su informe de 2010, encuentra que la mayor parte de la reducción de la pobreza y la convergencia de ingresos pueden explicarse por los procesos de desarrollo en China, y que si se sacara a China del cálculo, la situación se vería alterada radicalmente, lo cual plantea todo tipo de cuestionamientos que ameritan un examen mucho más detallado, y un análisis desde la perspectiva de los estudios críticos del desarrollo, podríamos añadir.

Una forma de avanzar con un estudio como tal - un primer paso tentativo-consiste en revisar la miríada de informes y estudios oficiales que se han preparado y publicado sobre los temas de inequidad y pobreza desde 2000. A continuación presentamos un breve esbozo de esa revisión.

Entre el 6 y el 9 de septiembre de 2000, la ONU organizó la Cumbre del Milenio para discutir su papel a principios del siglo XXI. En esta reunión, los líderes mundiales de 189 Estados miembros firmaron y ratificaron la Declaración del Milenio de las Naciones Unidas, que incluía la intención de ayudar a los ciudadanos en los países más pobres del mundo a fin de alcanzar una mejor vida en 2015. Esta pretensión quedó esbozada en la Declaración de los Objetivos de Desarrollo del Milenio (ODM), que incluía el objetivo (1)

1. Al final de la 53ra reunión anual del Consejo de Gobernadores del Banco Inter-Americano de Desarrollo (BID), realizada en Montevideo, del 14 al 19 de marzo de 2012, Alicia Bárcena, secretaria ejecutiva de CEPAL observó que ha llegado "el momento de la igualdad". Dijo que "en la actualidad, los responsables de la macroeconomía de la región están preocupados por la inequidad. Se trata de un gran cambio". Pero en cuanto a la naturaleza de esta preocupación, parecería tratarse más de un asunto de seguridad que de desarrollo. La inseguridad, vista como parte del problema de la inequidad social, es el principal desafío de América Latina, argumentó. 
de "erradicar la extrema pobreza y el hambre". Estos ODM constituyen una piedra de toque básica para una diversidad de informes sobre el progreso de la guerra contra la pobreza global — sobre acciones adoptadas por diferentes participantes y por agencias de desarrollo- realizadas durante más de una década por diferentes organizaciones internacionales dentro del sistema de la ONU.

\section{La cumbre del G8 en Okinawa:}

El reporte global de la pobreza, julio de 2000

La pobreza se hace notar en la síntesis ejecutiva del informe, "trasciende el ingreso". Con mayor detalle, aun cuando no muy precisamente, "incluye las dimensiones económica, social y de gobernanza". Los pobres, continúa la síntesis ejecutiva de la cumbre del G8 - haciendo eco de un tema elaborado en tres congresos del Banco Mundial entre 2002 y 2006 y del Informe mundial del desarrollo de 2006- "no son sólo quienes carecen de ingresos y recursos, sino también de oportunidades". Pero "los mercados y los empleos", continúa el reporte del G8 - dando voz a la concepción de los autores del informe y sus asesores en cuanto a las causas fundamentales de la pobreza- "con frecuencia son de difícil acceso debido a las bajas capacidades y a la exclusión geográfica y social". Aquí no se hace referencia, ni hay signo alguno en el propio informe, a la concepción dominante en la academia de que la pobreza es fundamentalmente estructural, arraigada en la estructura social del sistema y en las prácticas institucionalizadas que definen su "cultura" o, una vez más, como eventualmente sería reconocido y enfatizado por los autores del informe 2010 del Instituto de Investigación de las Naciones Unidas para el Desarrollo Social (UNRISD, por sus siglas en inglés) —en las "relaciones de poder [relaciones de poder entre los pobres y los ricos] que subyacen en el centro del desarrollo".

La "frágil posición" de los pobres, continúa la síntesis ejecutiva, "se exacerba por la inseguridad". Al vivir en "condiciones marginales, sin recursos de apoyo, los tropiezos resultan duros o imposibles de evitar". La situación de los pobres, concluye el informe, 
"empeora debido a la estructura de las sociedades e instituciones que tiende a excluir a los pobres de la participación en la toma de decisiones respecto a la dirección del desarrollo social y económico". Para concluir, la pobreza no está arraigada en la estructura social y económica de la sociedad, es decir, en el sistema, sino en la exclusión social y en la falta de oportunidades para el progreso de las personas por sí mismas (self-advancement), déficits que supuestamente son corregibles, y la falta de participación en la toma de decisiones, que no lo es.

El informe señala que a lo largo del último siglo se hicieron importantes mejoras en las áreas de la salud y educación, el progreso se reflejó en las tasas de mortalidad infantil en descenso, en un aumento en la expectativa de vida y en las tasas de alfabetismo; pero incluso así, se calcula que cerca de mil millones de personas viven todavía con menos de $\$ 1$ diario — la medida del Banco Mundial para la pobreza extrema, y unos 2.3 miles de millones con menos de $\$ 2$ diarios. Es decir, en conjunto unos 3.3 miles de millones de personas, aproximadamente dos quintas partes de la población mundial - la misma cifra que en 1973 cuando, por primera vez, los economistas del Banco Mundial "descubrieron" la pobreza global —-todavía son pobres a pesar de 37 años de guerra contra la pobreza global (o, según algunos, contra los pobres)-.

Banco Mundial (2000/01) Informe mundial del desarrollo: ataque a la pobreza

La gente pobre vive sin las libertades fundamentales de acción y decisión que los más acomodados toman por sentadas. Es frecuente que carezcan de alimento adecuado, vivienda, educación y salud, privaciones que evitan que tengan el nivel de vida que todo el mundo valora. También enfrentan una extrema vulnerabilidad ante las enfermedades, la dislocación económica y los desastres naturales. Es frecuente que estén expuestos al maltrato de las instituciones del Estado y la sociedad, y que carezcan de poder para influir en las decisiones clave que afectan sus vidas. 
Al comienzo del nuevo siglo, la pobreza sigue siendo un problema global de enormes proporciones. De los 6 mil millones de personas en el mundo (en 2000), 2.8 miles de millones viven con menos de $\$ 2$ diarios y 1.2 mil millones de ellas viven con menos de $\$ 1$ al día; ocho de cada 100 niños no vive hasta su quinto cumpleaños; nueve de cada 100 varones y 14 de cada 100 niñas que llega a la edad escolar no asiste a la escuela. La pobreza también es evidente en la falta de poder y de voz en el ámbito político de la gente pobre y en su extrema vulnerabilidad ante las enfermedades, la dislocación económica, la violencia personal y los desastres naturales. El flagelo del VIH/SIDA, la frecuencia y brutalidad de los conflictos civiles y las cada vez mayores disparidades entre los países ricos y el mundo subdesarrollado han incrementado el nivel de privación e injusticia.

El Informe del desarrollo mundial 2000//01, emitido por el Banco, que fue precedido por otros dos sobre pobreza (en 1980 y 1990), argumenta, empero, que sin duda son posibles reducciones considerables en todas estas dimensiones de la pobreza. Se requieren acciones en tres áreas complementarias:

- Promover oportunidades económicas para la gente pobre por medio de un crecimiento equitativo, mejor acceso a los mercados y recursos ampliados;

- Facilitar que la gente tenga poder haciendo que las instituciones del Estado respondan más ante las personas pobres y por la remoción de las barreras sociales que excluyen a las mujeres, los grupos étnicos y raciales, y a quienes están socialmente en desventaja, y

- Mejorar la seguridad al evitar las crisis económicas y al administrarlas bien cuando suceden, y proporcionando mecanismos para reducir las fuentes de vulnerabilidad que enfrenta la gente pobre. Pero las acciones de los países y las comunidades no serán suficientes. Se requerirán determinadas acciones globales para complementar las iniciativas nacionales y locales y lograr un máximo beneficio para la gente pobre en todo el mundo. 
Las acciones para ampliar la oportunidad, el empoderamiento y la seguridad pueden generar una nueva dinámica para el cambio que hará posible enfrentar la privación humana y crear sociedades comprometidas con la equidad y la justicia social —la ampliación de las opciones y oportunidades de las personas, y la realización de sus capacidades-, y aun así permanecer competitivas y productivas. Si el mundo en desarrollo y la comunidad internacional colaboran para abonar este nuevo entendimiento con recursos reales, tanto financieros como aquéllos encarnados en la gente y las instituciones — su experiencia, conocimiento e imaginación-, el siglo XXI verá un rápido progreso en la lucha contra la pobreza.

Además de estos "esfuerzos simultáneos [y concertados] por ampliar la oportunidad, la gestión y la seguridad", la clave para paliar la pobreza (si no es que una "nueva dinámica para el cambio") sigue siendo, en la visión del Banco, el desarrollo económico, que se consigue esencialmente a través de la liberalización del comercio y los mercados, la inversión en infraestructura, y la provisión de servicios sociales básicos a las personas pobres - de modo que se incremente su capital humano, para capacitar o darles poder para actuar y aprovechar sus oportunidades - para nivelar el campo de juego y proporcionar mayor igualdad de oportunidades.

Para concluir, el Banco argumenta que la evidencia confirma que se requiere el crecimiento de toda la economía (facilitado por las políticas "a favor del crecimiento", es decir, el neoliberalismo) para mejorar los ingresos de la gente pobre, lo que a largo plazo reducirá las dimensiones no monetarias de la pobreza. Además, desde la perspectiva del Banco, ampliar las capacidades humanas de la gente pobre - en lugar de realizar cambio estructural - sigue siendo un elemento central en cualquier estrategia de reducción de la pobreza. Esta perspectiva, podría añadirse, se contrapone con lo que podría ser vista como la perspectiva académica predominante en cuanto a la problemática de la pobreza. 
UNESCO (2002), CAPITAL SOCIAL Y REDUCCIÓN DE LA POBREZA: ¿QUÉ PAPEL ASIGNAR A ORGANIZACIONES DE LA SOCIEDAD CIVIL Y AL ESTADO?

El 28 de junio de 2000, en Ginebra, Suiza, se realizó el simposio internacional titulado "Formación de capital social en la reducción de la pobreza: ¿qué papel asignar a las organizaciones de la sociedad civil y al Estado?", organizado por el Programa de Gestión de las Transformaciones Sociales de la UNESCO (MOST, por sus siglas en inglés) y el Programa de Investigación Comparada sobre la Pobreza (CROP, por sus siglas en inglés) del Consejo Internacional de Ciencias Sociales. Se realizó en el marco de la 24 ta sesión especial de la Asamblea General respecto al resultado de la Cumbre Mundial para el Desarrollo Social e iniciativas posteriores, Ginebra (26 de junio al 1 de julio de 2000).

La Cumbre Mundial para el Desarrollo Social (Copenhague, marzo de 1995) abordaron tres temas centrales: erradicación de la pobreza; promoción del pleno empleo; y fomento de la integración social. En ellas se expresó el surgimiento de una determinación colectiva por tratar el desarrollo social como una de las más altas prioridades de las políticas nacionales e internacionales, y de ubicar al ser humano en el centro del desarrollo.

La 24ta sesión especial de la Asamblea General, titulada "Cumbre mundial para el desarrollo social y más allá: el logro del desarrollo social en un mundo en globalización", se realizó con tres objetivos: reafirmar la Declaración de Copenhague y el programa de acción adoptado en la Cumbre Mundial para el Desarrollo Social; identificar el avance logrado y las limitaciones encontradas, y recomendar acciones e iniciativas concretas para hacer avanzar los esfuerzos hacia una puesta en práctica plena y efectiva de los acuerdos alcanzados en la cumbre.

El Foro de Ginebra de 2000 se organizó en paralelo con la sesión especial de las Naciones Unidas. Fue diseñado para establecer un puente entre la sesión de la Asamblea General y el público en general. Atrajo a grupos parlamentarios, empresarios, sindicatos, 
académicos, organizaciones de la sociedad civil, organizaciones profesionales, así como gobiernos y organizaciones intergubernamentales. Fue en este marco que el simposio de MOST/CROP se organizó como una contribución al debate sobre las políticas y las estrategias para lograr la erradicación de la pobreza y lograr el desarrollo social.

Durante los cinco años transcurridos entre la Cumbre Mundial para el Desarrollo Social y 'Copenhague +5 ', se suscitaron varias deliberaciones acerca de temas que habían surgido en el debate internacional sobre el desarrollo antes, durante y después de la Cumbre Mundial para el Desarrollo Social. El papel del capital social fue uno de los temas debatidos en este contexto. Surgieron de las deliberaciones varias conclusiones y recomendaciones de importancia y éstas se reflejan en las iniciativas incluidas en el documento final de Ginebra, adoptado en la sesión especial de la asamblea general en Ginebra. En el documento también se hace referencia al fomento de la inversión en capital social. De tal modo, las Naciones Unidas y sus Estados miembros reconocen al capital social como un recurso importante para lograr las metas de la Declaración de Copenhague.

Muy aparte de esta Declaración, el concepto de capital social también es medular para un "nuevo paradigma" emergente de desarrollo local descentralizado en el cual el pobre se presenta como el "actor" líder, con poder para actuar por sí mismo en las localidades y comunidades de la sociedad rural (Atria et al., 2004). Este paradigma, al que Stiglitz había convocado ya desde 1998 en un influyente discurso (Stiglitz, 1998), aporta también las suposiciones y principios que se utilizan para construir un nuevo marco institucional y de políticas para el proceso de desarrollo.

Lo importante, y lo que discute Michael Woolcock en su contribución al informe de UNESCO, es la pregunta: ¿cómo lograr una reducción de la pobreza a través de la construcción de asociaciones entre Estados, sociedad civil y mercado? (Woolcock, 2002: 20). Otras preguntas que se abordan en el informe incluyen: ¿Qué papel asignar a la sociedad civil y al Estado? ¿Qué pueden hacer los actores civiles y las políticas para "movilizar recursos"? 
International Food Policy Research Institute (IFPRI) 2007:

Adoptar acciones a favor de las personas pobres y hambrientas del mundo

Una cumbre realizada en Beijing, en octubre de 2007, organizada por el International Food Policy Research Institute (IFPRI), a la que asistieron más de 400 diseñadores de políticas y otros interesados en el desarrollo internacional, se centró en la lucha contra la pobreza y en el avance logrado en realizar los ODM. El documento que saldría de esta cumbre, intitulado Taking Action for the World's Poor and Hungry People, reportó un avance considerable en la reducción de la tasa de pobreza global. La mayor parte de esta mejoría, a todas luces, puede ser o fue explicado por las altas tasas de crecimiento económico en China durante una década y media, lo que reanima un debate político de largo aliento en cuanto a los determinantes del crecimiento económico y las posibles compensaciones de la redistribución (crecimiento redistributivo/ crecimiento con equidad).

Hay que leer el informe para creerlo, y debería ser estudiado con ojo crítico. La principal conclusión puede sintetizarse brevemente: el mundo ha hecho avances considerables, habiendo reducido la incidencia de la pobreza extrema, desde $28.6 \%$ de la población en 1990 a $18.6 \%$ en 2004 ("con este ritmo, para 2015 se cumplirá el objetivo correspondiente en los ODM en cuanto a pobreza en el ámbito global). Sin embargo, el informe señala que el avance por regiones ha sido desigual: el "rápido crecimiento económico" en el este de Asia "ha beneficiado a todos los grupos casi igualmente", pero "la reducción de la pobreza se estanca en África subsahariana", dejando atrás a los "ultrapobres".

¿Quiénes son los ultrapobres? Más o menos, los crónicamente pobres: gente en áreas rurales remotas y marginales, sin acceso a los caminos, los mercados, y los servicios de educación y salud; gente que enfrenta exclusión debido a su género, etnia o discapacidad, es decir, minorías, y aquéllos que carecen de recursos, educación o acceso al crédito. 
Con base en estos hallazgos o esta teoría, el informe prescribe acciones que:

- Conlleven al "crecimiento incluyente", es decir, políticas neoliberales "a favor del crecimiento" y también "a favor de los pobres". Muchos críticos han argumentado que estas políticas constituyen un importante factor que contribuye a la pobreza global, generando nuevas formas de empobrecimiento y exacerbando las antiguas (Chossudovsky, 1997);

- Mejoren el acceso a recursos, mercados e infraestructura (servicios esenciales). Sobra mencionar que la pregunta no planteada es: ¿qué recursos: financiamiento, tierra, tecnología avanzada o nueva, un empleo digno? Para los agricultores de escala pequeña, campesinos con poca tierra o sin tierra, quienes constituyen una gran parte de los pobres en el ámbito rural, sigue vigente el tema crítico de la tierra y los medios para trabajarla productivamente, para lo cual el Banco Mundial recomienda un enfoque basado en el mercado ("market-assisted land reform"); ${ }^{2}$

- Aceleren las inversiones en salud, nutrición y educación, es decir, en el "capital humano", e

- "Incluyan a los excluidos".

UNDESA: Repensar la pobreza: informe de la situación social mundial, 28 de enero de 2010

En 1995, en Copenhague, los líderes globales que participaron en la Cumbre Mundial para el Desarrollo Social describieron la erradicación de la pobreza como un imperativo ético, político y económico, y la identificaron como uno de los tres pilares del desarrollo

2. El enfoque basado en el mercado para la reforma de la tenencia de la tierra que recomienda el Banco Mundial es bastante controvertido, tanto entre académicos como en las bases de los movimientos sociales formados por las organizaciones campesinas y las comunidades indígenas que soportan estos movimientos. Estos movimientos, por lo general, abogan por una reforma agraria encabezada por el Estado o, como en el caso del movimiento brasileño de los trabajadores rurales sin tierra, la acción directa. 
social. La erradicación de la pobreza desde entonces se ha convertido en el objetivo englobante del desarrollo, según se refleja en los objetivos de desarrollo acordados internacionalmente, incluyendo los ODM, que establecieron la meta de reducir a la mitad la pobreza extrema para 2015.

Rethinking Poverty, el Informe Sobre la Situación Social en el Mundo 2010 busca "contribuir a repensar la pobreza y su erradicación". Afirma: "la urgente necesidad de un cambio estratégico que se aleje del pensamiento fundamentalista del mercado, las políticas y prácticas de las décadas recientes hacia un desarrollo más sustentable y políticas orientadas a la equidad y adaptadas a las condiciones y circunstancias nacionales". Esas estrategias nacionales de desarrollo, mismas que fueron presentadas en el Cumbre Mundial de 2005, deben ser orientadas a la realización de los ODM. Y, declaran los autores, que "en el centro de este esfuerzo, se debe colocar políticas responsables de desarrollo y políticas macroeconómicas contracíclicas para promover inversiones productivas y generar empleo digno".

El informe resulta bastante convincente en cuanto a repensar la pobreza y los esfuerzos para la reducción de la pobreza, diciendo que una confianza extrema en las fuerzas del mercado y en la liberalización económica derivan en el descuido de las estrategias diseñadas nacionalmente y orientadas hacia el desarrollo, en detrimento de los pobres del mundo. La lección más importante es que los gobiernos necesitan desempeñar un papel en el desarrollo, integrando las políticas económicas y sociales que apoyan la producción incluyente y el crecimiento del empleo, a la vez que atacan la inequidad y promueven la justicia.

Naciones Unidas (2010):

Informe de los Objetivos de Desarrollo del Milenio (ODM)

Los esfuerzos colectivos hacia la realización de los ODM han logrado avances en muchas áreas. Las tendencias alentadores antes de 2008 habían puesto a muchas regiones en el camino de lograr al 
menos algunas de estas metas. El impulso del crecimiento económico en las regiones en desarrollo sigue siendo vigoroso y, al aprender de los muchos éxitos de incluso los países en mayor desventaja, lograr los ODM todavía se encuentra a nuestro alcance. Tal es el principal mensaje del informe de la ONU para 2010 sobre los ODM.

Según el informe, todavía se están logrando avances en la reducción de la pobreza, a pesar de los descalabros significativos a raíz de la recesión económica de 2008-2009 y a pesar de la crisis alimentaria y la de energía. Los países en desarrollo en su conjunto siguen en camino de lograr la meta de reducción de la pobreza para 2015. Se espera todavía reducir la pobreza al 15\% de la población para 2015, lo que se traduce en cerca de 920 millones de personas que viven debajo de la línea internacional de pobreza(1.25 \$dólares/día), la mitad de la cifra de 1990.

El informe enfatiza que, a pesar de que se ha logrado un avance, éste es desigual; y sin un gran impulso, muchas de los objetivos incluidos en los ODM probablemente no se logren en la mayor parte de las regiones. Los viejos y los nuevos desafíos amenazan con hacer más lento aún el progreso en algunas áreas o incluso echar por tierra algunos de los logros realizados hasta el momento. El número de personas que sufren de malnutrición sigue creciendo, mientras que el lento avance en reducir el hambre se ha estancado - o incluso se ha revertido- en algunas regiones entre $2000 \mathrm{y}$ 2002 , y entre 2005 y 2007. Aproximadamente, uno de cada cuatro niños de menos de cinco años de edad no cumple con su peso ideal, principalmente debido a falta de alimentos, la baja calidad de la comida que logran ingerir, la provisión inadecuada de agua y de servicios sanitarios y de salud, y las malas prácticas de cuidado y alimentación.

El reporte de los ODM estima que 1.4 miles de millones de personas todavía viven en pobreza extrema. Además, los efectos de la crisis financiera global probablemente persistan: las tasas de pobreza serán ligeramente superiores en 2015 e incluso después, hasta 2020, de lo que habrían sido si la economía mundial hubiera crecido de manera estable al ritmo anterior a la crisis. 
La equidad de género y el empoderamiento de las mujeres se encuentran en el centro de los ODM y constituyen precondiciones para superar la pobreza, el hambre y la enfermedad. Pero el avance ha sido lento en todos los frentes, desde la educación hasta el acceso a la toma de decisiones políticas.

Instituto de Investigación de las Naciones Unidas para el Desarrollo Social (UNRISD) (2010):

Combatir la pobreza y la desigualdad

El informe central de la UNRISD (UNRISD Flagship Report) constituye una importante iniciativa de investigación que se propone contribuir a los debates en torno a las nuevas estrategias políticas para la reducción de la pobreza en un marco posterior a los ODM. El informe se basa en gran medida en el Proyecto sobre Reducción de la Pobreza y Regímenes de Política, así como en la investigación pretérita del UNRISD sobre "Política social en un contexto de desarrollo (2000-2005)".

En septiembre de 2010, el UNRISD publicó su informe central sobre reducción de la pobreza, intitulado Combatir la pobreza y la inequidad, el cual resulta de una iniciativa de investigación de varios años en varios países sobre la efectividad de diferentes estrategias para reducir la pobreza. La principal tema abordado por el UNRISD es la persistencia de la pobreza global después de más de una década de esfuerzos combinados para lograr una reducción de la pobreza acorde con el primer ODM (reducir a la mitad la tasa de pobreza para el 2015): más de mil millones de personas continúan viviendo en condiciones de pobreza extrema, a pesar de los esfuerzos concertados de la comunidad internacional de desarrollo en apoyo del "nuevo desarrollismo" - el consenso en cuanto a la necesidad de "traer de vuelta al Estado" de modo que se generen las condiciones para una forma más incluyente de desarrollo (Osvaldo e Infante, 2009).

En contraste con las experiencias de países que tuvieron éxito históricamente en la reducción de la pobreza y la inequidad, 
las estrategias contemporáneas de reducción de la pobreza han "enfocado a los pobres" con una política de inclusión social y asistencia económica, sin que simultáneamente aborden las fuentes estructurales de la pobreza. El programa mexicano en contra de la pobreza, Oportunidades, constituye un excelente ejemplo. Estos acercamientos frecuentemente no toman en cuenta las dimensiones institucionales y políticas que resultan clave y que pueden ser a la vez causas de pobreza e inequidad, y obstáculos a su reducción. La investigación de UNRISD muestra, en cambio, que los avances efectivos se han dado principalmente a través de estrategias dirigidas por el Estado, estrategias que combinan objetivos de desarrollo económico con políticas sociales de tal manera que se apoyan recíprocamente en vez de ser contraproducentes (como en el caso de las políticas de "reforma estructural" aplicadas bajo el modelo neoliberal). También muestra de qué manera los resultados en términos de pobreza están configurados por complejas interconexiones de ideas, instituciones, políticas y prácticas en las esferas social, económica y política.

La investigación enfatiza los siguientes elementos centrales de una estrategia de desarrollo sustentable e incluyente: i) los patrones de crecimiento y de cambio estructural que generan y sostienen los empleos que son remunerados adecuadamente y accesibles para todos, independientemente de ingreso, estatus de clase, género o etnia; ii) las políticas sociales incluyentes basadas en derechos universales y que apoyan el cambio estructural, la cohesión social y la política democrática; iii) la protección de los derechos civiles, el activismo y los arreglos políticos que aseguran que los Estados respondan a las necesidades de los ciudadanos y que los pobres influyan en la manera en que se hacen las políticas; y iv) una política de inclusión social y las transferencias monetarias condicionadas a los pobres (por ejemplo, el programa Oportunidades en México o la Bolsa Familia de Brasil) no bastan. Para sostener los limitados logros de la guerra en contra de la pobreza global a lo largo de la década pasada es necesario abordar directamente la estructura de la inequidad social (la distribución 
social de la riqueza y el ingreso). Como argumentara la CEPAL (2010) en un reporte paralelo, y reiterara el RIMISP (2012) en un reporte de seguimiento, "es tiempo para la equidad". Para ser precisos, lo que se necesita es un cambio social sustantivo así como el abandono de las políticas (neoliberalismo) que sólo exacerban el problema.

\section{CONCLUSIÓN}

Desde la declaración de los ODM por la ONU en 2000, se ha dado un considerable avance en el cumplimiento de la meta de erradicar la pobreza absoluta y reducir sustancialmente la pobreza mundial en general (50\% para 2015), a través de acciones nacionales decisivas y cooperación internacional. $\mathrm{O}$, al menos, tal es la conclusión a la que llegan varios informes oficiales sobre la problemática de la pobreza y la guerra contra la pobreza global. Sin embargo, lo que no queda claro es la cuestión de agencia en la reducción de la pobreza. ¿Es resultado de acciones adoptadas por los gobiernos, con la cooperación internacional y la participación social - como sugieren varios reportes oficiales? ¿O es, en cambio, resultado de acciones llevadas a cabo por los mismos pobres? ¿O resultado de los cambios en la economía global? La respuesta a estas preguntas tendrá que esperar una revisión académica más plena; un examen más detallado y estudios posteriores de parte de sociólogos y economistas interesados en comprender las dinámicas de la problemática de la pobreza desde una perspectiva de los estudios críticos del desarrollo. Quedan varias cosas en claro: la pobreza está estrechamente relacionada y se basa en lo que la CEPAL describe como la "estructura de la inequidad social", y cualquier logro en el frente contra la pobreza no puede sustentarse en una política de inclusión social y transferencias directas a los pobres. Como declara la CEPAL en su informe central de 2010: "es tiempo para la equidad", es decir, un cambio social sustantivo en el sistema, las instituciones y las políticas que empobrecen y mantienen pobre a la gente (la 
globalización neoliberal). El propósito de la política pública (desarrollo equitativo) no debería ser sólo lograr una "igualdad de oportunidades" sino una "igualdad de condiciones". Esto requiere más que una reforma institucional y de política: requiere un cambio fundamental en un sistema sustentado por relaciones de poder de clase.

\section{REFERENCIAS}

Comisión Económica para América Latina y el Caribe (CEPAL) (2010), La hora de desigualdad: brechas por cerrar, caminos por abrir, Brasilia, CEPAL. ORGANISATION for Economic Co-operation and Development (OECD) (2010), Tackling Inequalities in Brazil, China, India and South Africa. The Role of Labour Market and Social Policies, París, OECD. (2011), Divided we stand: Why inequality keeps rising, París, OECD.

Centro Latinoamericano para el Desarrollo Rural (RIMISP) (2012), Poverty and Inequality: Latin American Report 2011, Santiago de Chile, RIMISP.

STIGLITZ, Joseph E. (1998), "More Instruments and Broader Goals: Moving Beyond the Post-Washington Consensus", Wider Annual Lectures, 2, Helsinki, WIDER.

SunKel, Osvaldo y Ricardo Infante (2009), Hacia un desarrollo inclusivo: El caso de Chile, Santiago, Chile, CEPAL.

UNITED Nations Department of Economic and Social Affairs (UNDESA) (2010), World Economic and Social Survey 2010. Retooling Global Development, Nueva York, ONU.

United Nations Development Programme (PNUD) (2010), Regional Human Development Report for Latin America and the Caribbean 2010, Nueva York, PNUD.

UNITED Nations Research Institute for Social Development (UNRISD) (2010), Combatiendo la pobreza y la desigualdad: cambio estructural, política social y la política, Ginebra, UNRISD.

Wolfensohn, James (1999), A Proposal for a Comprehensive Development Framework, Washington DC, World Bank, http://www.Worldbank.org/cdf/cdf-text.htm (10/10/2004). 